Olivier Landron, 1875-1970, L'Université catholique de l'Ouest. Enracinement et ouverture \& Catherine Masson, La Catho. Un siècle d'histoire de l'Université catholique de Lille 1877-1977

Paris, CLD éditions, 2012, 264 p.

\& Villeneuve-d'Ascq, Septentrion, 2011, 559 p.

\title{
Frédéric Gugelot
}

\section{OpenEdition}

Journals

Édition électronique

URL : http://journals.openedition.org/assr/25530

DOI : $10.4000 /$ assr.25530

ISSN : $1777-5825$

Éditeur

Éditions de l'EHESS

Édition imprimée

Date de publication : 30 décembre 2013

Pagination : 224

ISSN : 0335-5985

Référence électronique

Frédéric Gugelot, « Olivier Landron, 1875-1970, L'Université catholique de l'Ouest. Enracinement et ouverture \& Catherine Masson, La Catho. Un siècle d'histoire de l'Université catholique de Lille 1877-1977 ", Archives de sciences sociales des religions [En ligne], 164 | 2013, mis en ligne le 05 mars 2014, consulté le 21 septembre 2020. URL : http://journals.openedition.org/assr/25530 ; DOI : https:// doi.org/10.4000/assr.25530

Ce document a été généré automatiquement le 21 septembre 2020.

(c) Archives de sciences sociales des religions 


\section{Olivier Landron, 1875-1970,}

L'Université catholique de l'Ouest. Enracinement et ouverture \& Catherine Masson, La Catho. Un siècle d'histoire de l'Université catholique de Lille 1877-1977

Paris, CLD éditions, 2012, 264 p.

\& Villeneuve-d'Ascq, Septentrion, 2011, 559 p.

\section{Frédéric Gugelot}

\section{RÉFÉRENCE}

Olivier Landron, 1875-1970, L'Université catholique de l'Ouest. Enracinement et ouverture, Paris, CLD éditions, 2012, $264 \mathrm{p}$.

Catherine Masson, La Catho. Un siècle d'histoire de l'Université catholique de Lille 1877-1977, Villeneuve-d'Ascq, Septentrion, 2011, 559 p.

1 Bien que ces deux livres soient des œuvres de commande auprès de deux historiens " maison" comme le reconnaît Guy Bédouelle dans son introduction à l'ouvrage sur l'UCO (p. 19), ils apparaissent comme de solides bases historiographiques qui donnent accès à des archives inédites et offrent, quoique de façon inégale, bien des éléments de comparaison pour cerner le profil de ce pari catholique d'un enseignement supérieur confessionnel.

Ces ouvrages concernent deux des cinq universités catholiques fondées en France dans les années 1870, Angers et Lille. Leurs buts de fondation sont de faire "pénétrer dans toutes les matières de l'enseignement, dans les lettres et dans les sciences, les vrais 
principes de la foi » (Lettre de Philibert Vrau à Pie IX, Masson, p. 38) et de défendre la foi grâce à l'encadrement des jeunes pour les protéger des mauvaises influences de l'enseignement public. Le livre de Catherine Masson est une somme richement illustrée. L'apport iconographique est important, même si les sources sont rarement précisément indiquées. Les tableaux, cartes, images sont très nombreux tant sur les effectifs étudiants que sur le programme iconographique des bâtiments par exemple. Celui sur l'UCO ne comporte qu'un cahier photographique central de quatre pages essentiellement composé de portraits.

Ces deux universités partagent une chronologie comparable : une période de fondation largement impulsée par un milieu légitimiste et intransigeant (1875-1914), la consolidation entre les deux guerres, l'essor après 1945 (ils se fondent alors au sein de la Catho de Lille plus d'écoles tel l'IESEG, institut d'économie scientifique et de gestion, et l'EDHEC, école de hautes études commerciales du Nord, que dans les quatre-vingts ans précédents), les crises à partir des années 1960 et la refondation après 1970. Elles diffèrent profondément par leur taille. À la fin des années 1960, la Catho de Lille est la plus importante de France avec 6266 étudiants en 1968 dont 881 dans les facultés alors qu'Angers compte 941 étudiants de lettres en 1966, la moitié des effectifs de l'ensemble de l'UCO.

4 La question de l'identité catholique des enseignements est essentielle. Ces fondations sont définies comme des missions : à la fois dispenser un enseignement de qualité équivalent à celui de l'État, mais de le faire dans le cadre des principes de la foi catholique. Immédiatement on comprend les difficultés posées par ces conditions tant au niveau du recrutement des enseignants, de la délivrance des diplômes que sur les thématiques de recherche. Afficher son catholicisme fait partie intégrante du programme de fondation, ce catholicisme est perçu «comme un rempart contre certaines évolutions du monde moderne qui semblaient saper les fondements $d u$ message évangélique» (Landron, p. 27). Au même moment, bien des catholiques tentent de répondre à la sécularisation de la pensée en construisant une "science catholique » (François Laplanche), un savoir scientifiquement fondé et respectueux du dogme et des croyances. Ils veulent constituer une véritable alternative à la science réputée matérialiste à un moment où son magistère est croissant dans la société, d'autant qu'une "production catholique" même scientifique ne s'isole pas de sa réutilisation par des clercs et religieux dans un but apologétique. Ce projet "catholique » répond par une production scientifique au processus d'autonomisation des sciences ou des esthétiques (pour la littérature ou les arts sacrés). Il s'inscrit donc dans une tension entre la validation catholique accordée par l'Église et la reconnaissance scientifique désormais indépendante de l'institution ecclésiale. La fondation des universités catholiques s'inscrit dans ce contexte plus large de tentatives de construction d'une médecine catholique (voir Ruth Harris, Hervé Guillemain), d'une conception catholique du droit (voir Revue française d'histoire des idées politiques, 2008/2, $\mathrm{n}^{\circ}$ spécial « Les juristes catholiques »), d'une écriture littéraire catholique autour de la figure de l'écrivain catholique (Voir Alain Dierkens et alii, La croix et la bannière. L'écrivain catholique en francophonie, 2007). Angers est le projet personnel de Mgr Freppel, qui voulait contrebalancer la fondation parisienne, perçue par lui comme trop libérale autour de Mgr Dupanloup. La prière de Mgr Freppel pour la consécration à la Vierge de l'Université témoigne de cette orientation: «Faites, par votre glorieuse intercession, ô Mère admirable, que l'œil fixé, d'une part, sur la vérité de l'Immaculée Conception et, d'autre part, sur le Siège de Pierre qui par la bouche infaillible de Pie XI 
a défini cette vérité comme révélée par Dieu, que les élèves présents et futurs de l'Université d'Angers, combattent avec énergie et persévérance les erreurs qui contredisent le dogme » (Landron, p. 39). La faculté de théologie semble indispensable à l'existence d'une université catholique. Elle n'est pourtant pas première dans les fondations et elle est particulièrement surveillée. Ainsi en 1964, le cardinal Pizzardo, préfet de la Congrégation pour les séminaires et les universités catholiques, exige que le père Paul de Surgy soit éloigné de l'établissement. Deux ans plus tard, il en devient pourtant le doyen. Alors que les effectifs baissent fortement, le recteur Mgr Honoré et les institutions catholiques perçoivent la faculté de théologie comme "progressiste». Celle-ci s'engage d'ailleurs en faveur de la déconfessionnalisation de l'université (Landron, p. 196). De Surgy démissionne deux ans plus tard. La théologie disparaît de l'horizon d'identification des universités catholiques.

Des moments historiques apparaissent plus propices à l'essor des universités catholiques : la fondation se place dans un moment de la majorité monarcho-cléricale de l'Ordre moral dans les années 1870, puis Vichy qui donne un cadre juridique favorable à l'existence de ces universités et des subventions financières. Le régime du Maréchal Pétain reconnaît en effet l'utilité publique des universités catholiques de Lille, Angers, Lyon et Toulouse en 1941. Paris l'avait obtenu en 1940 (Masson, p. 358) et Angers reçoit des subventions d'État entre 1942 et 1944 (Landron, p. 102). Un troisième moment ne figure pas dans les deux ouvrages, qui s'arrêtent en 1970 pour Angers et 1977 pour Lille. En 2009, le président Sarkozy signe un décret très favorable à la reconnaissance des grades et diplômes des universités catholiques au moment où l'État et le Ministère de l'Enseignement supérieur et de la Recherche versent, en 2011, plus de 42 millions d'euros aux institutions d'enseignement privé catholique. Le diplôme et l'argent sont en effet les principales difficultés auxquelles elles se heurtent au cours de leur siècle d'existence.

6 Le grand souci des universités catholiques est la quête incessante d'argent. La souscription de naissance permet à Lille la collecte de six millions et demi de francs auprès de huit mille donateurs, dont deux mille membres du clergé. Des campagnes de soutiens sont organisées, des clercs se spécialisent dans le ratissage des paroisses à l'exemple à Angers des Missionnaires de l'UCO qui prêchent dans les paroisses du Grand Ouest (Landron, 132-133). En 1959, leur action permet de récolter six millions de francs sur un budget total de soixante millions. À Lille, le vice-recteur Henri Barbeau lance des "Campagnes d'information et de propagande " pour promouvoir la Catho avec une tournée des collèges privés de la région Nord et, dès mai 1948, il visite pendant un mois une moitié de la France puis l'autre l'année suivante, et cela, pendant douze ans. Ces universités bénéficient de l'appui des milieux économiques et, de ce point de vue, une vraie différence existe dès l'origine entre Lille et Angers. Le terreau économique du Nord, et en particulier le soutien constant et important de la famille Feron-Vrau, qui pendant trente ans garantit le financement de l'institution, permet à Lille de disposer de ressources conséquentes et continues bien plus importantes que celle d'Angers. D'autant que l'implantation à Angers d'une Université d'État en 1971 limite les subventions des collectivités territoriales qui, avec les droits d'inscription et le produit des quêtes, sont les principales ressources. Néanmoins, «nous vivons d'expédients sans lesquels il nous faudrait fermer les portes ", avoue André Sander, le président du CA de Lille entre 1954 et 1960 (Masson, p. 433). L'évêque d'Arras, Mgr Huyghe, dénonce dans une lettre au recteur, Mgr Leclercq, des opérations qui «se moquent de l'honnêteté " pour faire face aux difficultés financières (Masson, p. 456). Angers souffre de l'absence 
d'un réel soutien du clergé angevin et encore plus breton. Le rattachement des diocèses bretons, contre leur volonté, à Angers plutôt qu'à Paris, est une lutte de tous les instants. L'assise lilloise est aussi de ce point de vue plus solide comme le concrétise la création d'un diocèse à Lille en 1913.

7 Les établissements dépendent donc largement de l'appui des fidèles pour leur financement. Le soutien des familles catholiques n'est néanmoins pas acquis. Ces facultés sont constamment confrontées à la question de la reconnaissance des formations, elles peuvent délivrer des diplômes canoniques non reconnus, et/ou opter pour des diplômes d'État dont celui-ci a le monopole. Dans ce cas, elles doivent faire valider leurs formations par des universités publiques ou des jurys rectoraux selon les époques. Or les familles doutent de la qualité des enseignements et se heurtent à l'absence ou aux difficultés de délivrance des diplômes d'État. Ce refus de la collation des grades freine le recrutement. Bien des familles et des étudiants catholiques choisissent l'Université d'État, pourtant "plus exigeante» (Masson, p. 267), car elle détient la délivrance des diplômes. Ainsi la faiblesse de leur formation et les réticences de l'Université d'État de Lille imposent aux étudiants de médecine de la Catho de passer leur examen à Nancy. Au moment de la crise des équivalences de 1969, des étudiants sont contraints de repasser une deuxième fois des examens. Ils manifestent et certains participent à une grève de la faim (Landron, p. 226-231 - Masson, p. 469-475).

ux fondations sont confrontées au difficile recrutement des étudiants mais aussi des enseignants. La question salariale est constante. À Angers comme à Lille, les enseignants constatent la réelle différence de traitement avec le public. Il est vrai que longtemps les formateurs cléricaux ont assuré une main-d'œuvre peu couteuse et corvéable à merci. Angers compte quarante ecclésiastiques sur cinquante-deux enseignants à la faculté des lettres de 1870 à 1940. Ils cumulent d'ailleurs de nombreux enseignements. Mais le besoin de recruter des chercheurs de qualité et des enseignants de référence se heurte aux bas salaires. Ainsi l'UCO n'hésite pas à tricher sur le nombre de doctorants qui enseigne les sciences afin de répondre aux contraintes imposées par l'État pour justifier l'ouverture d'une faculté de sciences, des professeurs fictifs sont aussi nommés (Landron, p. 75-76: "prêts de titres de docteurs»). Si l'argument catholique joue parfois et permet d'imposer des sacrifices, l'attrait du public reste fort. Les autorités s'inquiètent donc de façon récurrente du faible engagement des enseignants, du turn-over des professeurs, de l'insuffisance des publications. La solution est un fort recrutement endogame au sein en particulier des anciens élèves. Il existe, comme dans le public, des dynasties professorales : à Angers, Ferdinand HervéBazin voit son beau-frère et ses fils le rejoindre. Pour les étudiants, leurs origines sont largement régionales. Angers comme Lille sont d'abord des "université[s] de proximité »(Landron, p.107-121). Ces deux établissements ne pourraient d'ailleurs survivre sans les recteurs, les clercs et religieuses, les animateurs, les enseignants, les étudiants aussi. Des figures se détachent, Mgr Freppel évidemment pour Angers ou Norbert Segard pour Lille. La somme d'engagements et de dévouements que cristallisent la fondation et le développement de ces universités catholiques, que l'on retrouve dans nombre d'autres associations, syndicats, mouvements, est un trait et une force $d u$ catholicisme français $a u x^{e}$ siècle. Sa capacité mobilisatrice peut ainsi orienter vers de tels projets à la fois financement et énergie.

9 La crise des années 1960 n'épargne pas les universités catholiques. Malheureusement, Catherine Masson passe très rapidement et Olivier Landron n'use que du prisme de la 
crise générale de l'autorité, qui ne pouvait que remettre en cause les modes de fonctionnement de ces institutions, pour évoquer les événements de 1968. On regrette que seules les réactions de Mgr Honoré nous permettent de saisir l'ampleur et les formes de la crise à Angers. D'autres sources, d'autres témoignages auraient peut-être permis de nuancer ou de contredire la vision du recteur. Les universités catholiques avaient déjà été confrontées aux phénomènes de politisation avec l'Action française dans les années 1920 avant le "gauchisme" dans les années 1960. Les autorités s'opposent dès le début des années 1960 à ce qu'ils appellent la "politisation » des étudiants, qui ne concerne sur le plan actif souvent qu'une minorité. Une contestation estudiantine de gauche se développe en effet autour de la Guerre d'Algérie. En 1962, une grève se déclenche contre les attentats de l'OAS. Le mode de gouvernance, la confessionnalisation des établissements sont contestés. À chaque fois, les autorités tentent d'en limiter l'expression : «La politique distrait et divise. Les étudiants doivent s'abstenir d'apporter les préoccupations politiques dans les études qui ne peuvent qu'en souffrir. » (Instruction épiscopale du 16 janvier 1928, Masson, p. 314). En fait le courant des catholiques sociaux et des démocrates-chrétiens est plus facilement accueilli à Lille. Les engagements de nombreux enseignants et les options des évêques du Nord sont très favorables aux problématiques de l'Action catholique et la Catho est un vivier de militants chrétiens.

10 Ces deux universités trouvent des solutions salvatrices proches qui passent essentiellement par la multiplication des écoles ou instituts. Elles démontrent une réelle réactivité par rapport aux demandes économiques et sociales. Lille fonde dès 1924 une école de journalisme, la première de France, qui devient indépendante en 1960. Les liens avec le tissu économique local sont forts. Dès 1895, Lille ouvre un Institut technique roubaisien qui au départ forme des techniciens supérieurs textiles et à partir de 1935 aussi des ingénieurs. En 1956, Thomson-Houston implante une usine à Angers au moment même où l'UCO ouvre une École supérieure d'Électronique de l'Ouest, la même année d'ailleurs que Lille. Elle ouvre en 1961 une quatrième année qui oriente vers le titre d'ingénieur alors qu'est annoncée l'ouverture en 1963 de l'usine Bull-Anjou. Cette réactivité par rapport aux demandes professionnelles explique l'inventivité et la multiplication des formations qui ouvrent aussi facilement qu'elles ferment. La faculté de droit d'Angers, la première à ouvrir à l'origine avant les lettres, sciences et théologie, ferme en 1968 faute de financement alors que celle de Lille est mise en sommeil en 1961 et ne rouvre qu'en 1993. Angers compte dès 1898 une École supérieure d'agriculture et en 1909 une École supérieure de commerce. Lille ouvre une école des Hautes Études industrielles dès 1885, des Hautes Études agricoles dès 1887-1897. Après une interruption, l'ISA (Institut supérieur d'agriculture) est créé en 1963. L'Université d'Angers s'est profondément transformée en instituts professionnalisants au point que s'estompe l'aspect universitaire classique. Le 29 mai 1970, l'ensemble des enseignants est licencié, 120 personnes dont 80 prêtres. Cette action solde la crise de 1968, des contestataires ne sont pas réembauchés et des clercs sont éloignés tel le père Joseph Traineau (Landron, p. 240). Le 21 octobre, l'UCO rouvre sous la forme de quatre instituts : Formation des professeurs, Mathématiques, Langues vivantes et Psychologie sociale appliquée. Seule subsiste la faculté de théologie. Les étudiants de la FEUCO s'en inquiètent dès 1964 : «Il faut aussi noter l'autonomie que les écoles prennent de plus en plus vis-à-vis de l'Université catholique au point que certaines d'entre elles tendent à s'en détacher complètement. Cet état de fait peut, à l'avenir, modifier totalement leur orientation: les écoles risquent, en effet, de se limiter à former des techniciens 
auxquels il manquera la dimension universitaire et chrétienne. » (Landron, p. 212-213). Si la déconfessionnalisation ne se produit pas au niveau des titres et des structures, elle se réalise largement par l'acquisition de l'autonomie des écoles et instituts périphériques. La dimension spirituelle et morale des universités catholiques s'estompe avec leur prise de distance administrative et financière. Ces deux universités connaissent un identique processus de décléricalisation que symbolise l'élection en 1970 du premier directeur laïc de l'ÉSA d'Angers et l'arrivée d'un recteur laïc, en 1979 à la Catho de Lille. Ce processus est aussi celui d'une dissociation lente des formations intellectuelles et chrétiennes pourtant au cœur du projet d'origine des universités catholiques. La transformation d'Angers en instituts et la création à Lille d'une structure fédérative, la Fédération universitaire et polytechnique de Lille, ambitionnent de sauvegarder ces universités tout en reconnaissant le développement des écoles alors que les facultés déclinent. Elles marquent néanmoins la mise en sommeil du projet catholique.

11 Nées dans un même contexte, sous une même impulsion, les universités catholiques d'Angers et de Lille évoluent selon des traits proches, à des moments identiques et sous les mêmes contraintes (financement, validation catholique, recrutement des enseignants et des étudiants...). Leur destin est pourtant bien différent. Angers est constamment en difficulté et ne parvient pas à subsister comme une université alors que, malgré les soubresauts, la Catho de Lille maintient et développe sa vocation universitaire. Pourtant aux origines, Angers est sans concurrence, car il faut attendre 1971 pour qu'une université d'État s'installe dans la même ville. Le problème est donc bien celui du bassin de recrutement et de rayonnement d'autant qu'elles dépendent largement de la générosité des catholiques. 